\title{
INFLUÊNCIA DOS PRECURSORES DE PRATA NO CRESCIMENTO DE NANOPARTÍCULAS METÁLICAS EM VIDROS ÓXIDOS DE METAIS PESADOS
}

\author{
Paula S. Valle, Murilo Montesso e Marcelo Nalin* \\ Departamento de Química - Universidade Federal de São Carlos, CP 676, 13565-905 São Carlos - SP, Brasil \\ José Pedro Donoso, Igor D. A. Silva e Claudio J. Magon \\ Instituto de Física de São Carlos - Universidade de São Paulo, CP 369, 13560-970 São Carlos - SP, Brasil
}

Recebido em 21/9/12; aceito em 11/4/13; publicado na web em 4/6/13

\begin{abstract}
INFLUENCE OF SILVER PRECURSORS ON GROWTH OF METALLIC NANOPARTICLES IN HEAVY METAL OXIDE GLASSES. In this work we report a systematic study on the influence of the chemical nature of silver precursors on the formation of glass-ceramics from oxide glasses. Thermal, structural and optical properties were analyzed as a function of the glass composition. Controlled crystallization was achieved by thermal treatment of the samples above glass transition. The influence of time of treatment on both nanoparticle growth and optical properties of the samples was studied by transmission electron microscopy and UV-Vis spectroscopy, respectively. Results showed that only glasses containing $\mathrm{AgCl}$ and $\mathrm{AgNO}_{3}$ led to glass-ceramics growth after thermal treatment.
\end{abstract}

Keywords: glasses; glass-ceramics; nanoparticles.

\section{INTRODUÇÃO}

Vidros óxidos contendo metais pesados (VOMP) têm sido bastante estudados para aplicações em óptica por apresentarem altos valores de índice de refração linear e não linear. ${ }^{1}$ Esta propriedade advém da hiperpolarizabilidade dos átomos pesados presentes na matriz vítrea. Além dos efeitos não lineares, outras propriedades, como baixa absorção de dois fótons e efeito Kerr ultra-rápido, classificam estes materiais como candidatos a chaveadores ou fibras ópticas para aplicações no campo da fotônica apresentando grande interesse tecnológico. ${ }^{1}$ Com intuito de explorar mais amplamente estas propriedades, muitos trabalhos vêm sendo dedicados a incorporação de metais nobres, tais como $\mathrm{Ag}, \mathrm{Au}$, $\mathrm{Pt}$ e $\mathrm{Cu}$, em composições de VOMP. ${ }^{2}$ Utilizando processos de tratamento térmico ${ }^{3}$ ou irradiação com lasers ${ }^{4}$ (fotorredução), pode-se induzir o crescimento destes metais na forma de nanopartículas. Vidros contendo nanopartículas cristalinas são denominados de nano-vitrocerâmicas. ${ }^{5}$ Outras técnicas também podem ser empregadas para incorporação de íons metálicos e subsequente preparação de nanocristais em vidros, tais como troca iônica ou implantação de íons negativos, ${ }^{6,7}$ porém, em ambos os casos, uma etapa adicional de tratamento térmico também é necessária.

Vidros estão entre os melhores hospedeiros para nanopartículas de metais nobres e apresentam interessantes aplicações em fotôni$\mathrm{ca}^{8}$ devido, principalmente, à presença do efeito de ressonância de plasmons superfíciais (SPR). Este fenômeno, proveniente de efeitos quânticos nos nanocristais metálicos, pode ser explicado como oscilações coletivas de elétrons na superfície das nanopartículas ${ }^{9}$ e vem sendo utilizados em dispositivos plasmônicos ${ }^{2}$ e sensores. ${ }^{10}$ Embora muitos trabalhos científicos já tenham demonstrado o potencial de aplicações das vitrocerâmicas contendo nanopartículas de prata, não foi encontrado nenhum trabalho com viés fundamental, focando, principalmente, na influência dos diferentes precursores de prata nas propriedades finais dos materiais, bem como, no crescimento das nanopartículas. Dessa maneira, neste trabalho analisou-se a influência da incorporação de diferentes precursores de prata nas propriedades térmicas, estruturais e ópticas de vidros óxidos de

*e-mail: mnalin@ufscar.br metais pesados. Também é mostrado como o precursor influencia no crescimento de nanopartículas metálicas utilizando a metodologia de tratamento térmico. $\mathrm{O}$ sistema vítreo utilizado para análise foi o $\mathrm{SbPO}_{4}-\mathrm{WO}_{3}-\mathrm{PbO}-\mathrm{X}$, tendo que $\mathrm{X}$ pode ser $\mathrm{AgCl}, \mathrm{AgO}, \mathrm{Ag}_{2} \mathrm{O}$ ou $\mathrm{AgNO}_{3}$. As amostras foram estudadas por calorimetria exploratória diferencial (DSC), difração de raios X, espectroscopias UV-Vis, Raman e M-Lines, ressonância paramagnética eletrônica e microscopia eletrônica de transmissão.

\section{PARTE EXPERIMENTAL}

\section{Preparação das amostras vítreas}

Foram preparadas quatro amostras com base no sistema vítreo $1-\mathrm{x}\left(0,5 \mathrm{WO}_{3}-0,3 \mathrm{SbPO}_{4}-0,2 \mathrm{PbO}\right)-\mathrm{x}$, com x representando $\mathrm{AgCl}$, $\mathrm{AgNO}_{3} \mathrm{AgO}$ e $\mathrm{Ag}_{2} \mathrm{O}$. As concentrações dos compostos de prata utilizados totalizaram $5 \%$ em mol de $\mathrm{Ag}$. Todos os reagentes de prata, bem como o $\mathrm{WO}_{3}$ e $\mathrm{PbO}$, possuem pureza analítica. $\mathrm{SbPO}_{4}$ foi preparado segundo o procedimento descrito por Nalin e colaboradores. ${ }^{11}$ Os componentes dos vidros foram pesados e macerados em um almofariz de ágata, em seguida transferidos para um cadinho de platina e levados ao forno. Para as amostras de $\mathrm{AgCl}$ e $\mathrm{AgO}$, realizou-se a fusão a $1100{ }^{\circ} \mathrm{C}$ durante $30 \mathrm{~min}$. Posteriormente, a temperatura do forno foi reduzida a $980^{\circ} \mathrm{C}$, onde o líquido permaneceu por 5 min antes de ser transferido para um molde de aço, previamente aquecido a $350{ }^{\circ} \mathrm{C}$. As amostras contendo $\mathrm{Ag}_{2} \mathrm{O}$ e $\mathrm{AgNO}_{3}$ foram fundidas a $1100{ }^{\circ} \mathrm{C}$, porém, a temperatura de transferência para o molde foi de $1010^{\circ} \mathrm{C}$. As amostras foram deixadas no forno para recozimento durante $2 \mathrm{~h}$ à $350{ }^{\circ} \mathrm{C} \mathrm{e}$, posteriormente, o forno foi desligado e resfriado até a temperatura ambiente. As amostras foram então cortadas e polidas usando lixas de $\mathrm{SiC}$ com diferentes granulometrias.

\section{Tratamento térmico e crescimento das nanopartículas}

A formação das vitrocerâmicas foi realizada por tratamento térmico a $480{ }^{\circ} \mathrm{C}$ por diferentes tempos $(5$ e $10 \mathrm{~h}$ ). Para minimizar efeitos secundários e evitar possíveis variações nas propriedades das amostras durante o processo de crescimento das nanopartículas, os 
tratamentos térmicos foram realizados utilizando-se uma amostra de cada sistema em um único procedimento.

\section{Caracterização das amostras}

Os materiais foram caracterizados visando a estudar possíveis variações nas propriedades estruturais, ópticas e térmicas em função da adição dos compostos de prata. As temperaturas características dos vidros $\left(T_{g}\right.$, transição vítrea e $T_{x}$, início da cristalização) foram obtidas usando Calorimetria Exploratória Diferencial (DSC) em um equipamento 2910 da TA Instruments, na faixa de temperatura entre 200 e $600{ }^{\circ} \mathrm{C}$, usando pedaços de vidros ( $\left.30 \mathrm{mg}\right)$, em panelinhas de alumínio, seladas e sob atmosfera de $\mathrm{N}_{2}$. A taxa de aquecimento utilizada foi de $10{ }^{\circ} \mathrm{C} \mathrm{min}^{-1}$. O erro estimado utilizando esta técnica é de $\pm 2{ }^{\circ} \mathrm{C}$ tanto para $\mathrm{T}_{\mathrm{g}}$ como para $\mathrm{T}_{\mathrm{x}}$. Difração de raios $\mathrm{X}$ foi empregada para analisar a presença de fases cristalinas existentes nas amostras vítreas antes e após o tratamento térmico e foi realizada pelo método de pó, utilizando um difratômetro Simens Krystalloflex com radiação Cuк, no intervalo de 2 , entre 04 e $70^{\circ}$. Espectros de espalhamento Raman foram obtidos usando um espectrômetro HORIBA (Jobin Yvon) - LabRAM-HR equipado com um microscópio (micro Raman) e laser de He-Ne, com l = 632,8 nm, no intervalo de $100 \mathrm{a}$ $1400 \mathrm{~cm}^{-1}$. As curvas na região do UV-Vis foram obtidas dos vidros polidos usando um equipamento VARIAN, modelo Cary 5000 Scan, na faixa espectral de 2000 a $400 \mathrm{~nm}$. Os valores de índice de refração foram obtidos utilizando um equipamento MATRICON $2010 \mathrm{com} \lambda$ $=632 \mathrm{~nm}$, sendo o erro associado à medida de $10^{-4}$. Para confirmar a presença de nanopartículas foi utilizado um microscópio eletrônico de transmissão (MET) Philips CM 120. A preparação da amostra foi feita primeiramente, cominuindo-se um pedaço do vidro em um almofariz. O pó foi suspenso em etanol utilizando um banho ultrassônico e, posteriormente, gotas da suspensão foram depositadas sobre uma grade de cobre recoberta com filme de carbono.

As amostras vítreas foram caracterizadas por ressonância paramagnética eletrônica (RPE) a partir dos vidros pulverizados antes do tratamento térmico, utilizando um espectrômetro Varian E-109 operando na banda-X $(9,476 \mathrm{GHz})$. As medidas foram realizadas a $\mathrm{T}=-258{ }^{\circ} \mathrm{C}$ e à temperatura ambiente. É importante mencionar que as medidas de EPR foram realizadas com todas as amostras nas mesmas condições experimentais, ou seja, mesma temperatura (-258 $\left.{ }^{\circ} \mathrm{C}\right)$, mesma potência de microondas $(1 \mathrm{~mW})$ e mesma modulação $(5$ $\mathrm{G})$. As intensidades dos espectros foram corrigidas pelas massas das amostras e, para fazer a comparação entre eles, normalizamos pela intensidade da linha do $\mathrm{Fe}^{3+}$ que está presente como impureza natural em todas as amostras. Esse estudo foi realizado visando subtrair o efeito das impurezas na medida permitindo identificar o sinal da prata em $g=2,07$. Posteriormente os valores obtidos para a matriz pura foi subtraído dos dados obtidos para as amostras contendo os compostos de prata.

\section{RESULTADOS E DISCUSSÃO}

\section{Caracterização dos vidros sem tratamento térmico}

Como dito na sessão experimental, os vidros contendo $\mathrm{AgCl}$ e $\mathrm{AgO}$ foram obtidos em temperaturas diferentes daqueles contendo $\mathrm{Ag}_{2} \mathrm{O}$ e $\mathrm{AgNO}_{3}$. A maior temperatura antes do vertimento do líquido no molde pré-aquecido se deve ao fato que as amostras contendo $\mathrm{Ag}_{2} \mathrm{O}$ e $\mathrm{AgNO}_{3}$ se tornavam muito viscosas abaixo de $1010{ }^{\circ} \mathrm{C}$, dificultando a vazão do fundido para o molde. Independentemente do composto de prata adicionado ao vidro, todas as amostras obtidas são transparentes e apresentam coloração amarela. Difratogramas de raios $\mathrm{X}$ foram obtidos para as composições vítreas e, em todos os casos, somente a presença de halos de difração, característicos de materiais não-cristalinos foram observados. A influência da natureza química do composto de prata nas temperaturas características dos vidros foi analisada por DSC. As curvas obtidas para as amostras sem tratamento térmico acima de Tg são mostradas na Figura 1a. Os valores das temperaturas características foram comparados com aqueles obtidos para um vidro sem adição de prata $(\mathrm{Tg}=459$ e $\mathrm{Tx}$ $\left.=579{ }^{\circ} \mathrm{C}\right) .{ }^{12}$ Como podemos observar, a incorporação dos diferentes compostos de prata aumenta ligeiramente o valor de Tg para a amostra contendo $\mathrm{Ag}_{2} \mathrm{O}$, entretanto, para as demais composições Tg permanece constante. Por outro lado, podemos observar que a adição dos óxidos aumenta significativamente a temperatura de Tx. O parâmetro Tx-Tg é comumente utilizado para estimar a estabilidade térmica de uma composição vítrea frente à cristalização. Assim, podemos afirmar que a adição dos óxidos aumenta a estabilidade dos vidros em, pelo menos, $50^{\circ} \mathrm{C}$ quando comparado às composições contendo $\mathrm{AgCl} \mathrm{e}$ $\mathrm{AgNO}_{3}$. Os valores experimentais de DSC são resumidos na Tabela 1.

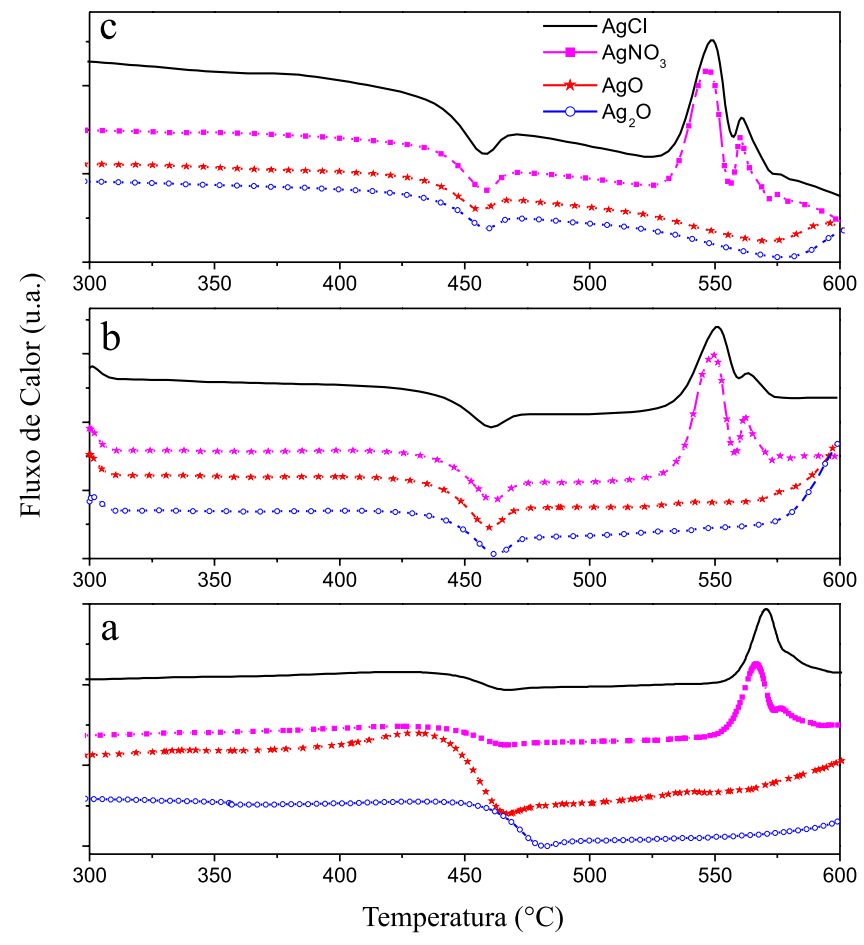

Figura 1. Curvas de DSC dos vidros contendo diferentes precursores de prata: a) sem tratamento térmico, b) após $5 \mathrm{~h} \mathrm{e} \mathrm{c)} \mathrm{após} 10 \mathrm{~h} \mathrm{a} 480^{\circ} \mathrm{C}$

Os compostos de prata incorporados aos vidros podem sofrer decomposição durante o processo de fusão a alta temperatura segundo as reações abaixo:

$$
\begin{aligned}
& 2 \mathrm{AgCl}_{(s)} \stackrel{\Delta}{\longrightarrow} 2 \mathrm{Ag}_{(s)}^{0}+\mathrm{Cl}_{2(g)} \\
& 2 \mathrm{AgNO}_{3(s)} \stackrel{\Delta}{\longrightarrow} 2 \mathrm{Ag}_{(s)}^{0}+2 \mathrm{NO}_{2(g)}+O_{2(g)} \\
& 2 \mathrm{Ag}_{2} \mathrm{O}_{(s)} \stackrel{\Delta}{\longrightarrow} 4 \mathrm{Ag}_{(s)}^{0}+\mathrm{O}_{2(g)} \\
& 4 \mathrm{AgO}_{(s)} \stackrel{\Delta}{\longrightarrow} 2 \mathrm{Ag}_{2} \mathrm{O}_{(s)}+\mathrm{O}_{2(g)} \stackrel{\Delta}{\longrightarrow} 4 \mathrm{Ag}_{(s)}^{0}+\mathrm{O}_{2(g)}
\end{aligned}
$$

Nesse sentido, todos os compostos levam ou deveriam levar à formação de prata metálica nas matrizes vítreas durante o procedimento de preparação das amostras. Assim, devemos considerar que a prata deve estar na forma de clusters, contendo somente alguns átomos metálicos e com dimensões muito reduzidas, uma vez que não foi possível observar a presença de nanopartículas por microscopia eletrônica nestas amostras. A confirmação da formação de 
Tabela 1. Temperaturas características obtidas para as amostras contendo diferentes compostos de prata e tratadas durante diferentes períodos

\begin{tabular}{|c|c|c|c|c|c|c|c|c|c|}
\hline \multirow{2}{*}{ Compostos } & \multicolumn{3}{|c|}{ SN } & \multicolumn{3}{|c|}{ TT-5h } & \multicolumn{3}{|c|}{ TT-10h } \\
\hline & $T g$ & $T x$ & $T x-T g$ & $T g$ & $T x$ & $T x-T g$ & $T g$ & $T x$ & $T x-T g$ \\
\hline $\mathrm{AgCl}$ & 444 & 559 & 118 & 441 & 530 & 89 & 438 & 532 & 94 \\
\hline $\mathrm{AgNO}_{3}$ & 442 & 554 & 113 & 443 & 533 & 90 & 442 & 532 & 90 \\
\hline $\mathrm{AgO}$ & 447 & - & $>169$ & 437 & 579 & 142 & 438 & 575 & 137 \\
\hline $\mathrm{Ag}_{2} \mathrm{O}$ & 463 & - & $>137$ & 437 & 578 & 141 & 438 & 580 & 142 \\
\hline$S A g *$ & 459 & 579 & 120 & - & - & - & - & - & - \\
\hline
\end{tabular}

prata metálica foi acompanhada usando RPE. Os espectros de RPE da Figura 2 apresentam três sinais. A ressonância em 1570 Gauss $(g=4,3)$ é atribuída a íons $\mathrm{Fe}^{3+}$ isolados em simetria tetraédrica ou octaédrica com distorção rômbica. ${ }^{15,16} \mathrm{O}$ íon $\mathrm{Fe}^{3+}$ tem configuração eletrônica $3 \mathrm{~d}^{5}$, com $\operatorname{spin} S=5 / 2$ no estado fundamental ${ }^{6} \mathrm{~S}_{5 / 2}$. Na região de $g »$ 2,0 (em torno de 3400 Gauss na Figura 2), o espectro de RPE mostra dois sinais superpostos, sendo um deles composto por um conjunto de linhas de pouca intensidade, o qual é característico do íon paramagnético $\mathrm{Mn}^{2+}$ ocupando sítios com simetria octaédrica com distorção tetragonal. ${ }^{17} \mathrm{O}$ íon $\mathrm{Mn}^{2+}$ tem configuração eletrônica $3 \mathrm{~d}^{5}$ e spin eletrônico $S=5 / 2$. Como o spin nuclear do isótopo ${ }^{55} \mathrm{Mn}$ (abundância natural $100 \%$ ) é $I=5 / 2$, o espectro de RPE do $\mathrm{Mn}^{2+}$ consiste em um conjunto de seis linhas hiperfinas. Esta estrutura hiperfina resulta da interação dipolo-dipolo entre o momento magnético do núcleo ${ }^{55} \mathrm{Mn}$ e o momento eletrônico do elétron desemparelhado do $\mathrm{Mn}^{2+}$. A separação entre estas linhas no espectro permite determinar a constante hiperfina, $A=80 \pm 6$ Gauss, valor que está de acordo com o relatado para $\mathrm{Mn}^{2+}$ em vidros óxidos. Cabe salientar que estes dois sinais, em $g=4,3$ e $g$ » 2,0, estão presentes também nas amostras de vidro não dopadas, sugerindo que eles resultem da presença de impurezas, $\mathrm{Fe}$ e Mn, nos óxidos metálicos utilizados na preparação dos vidros. O sinal intenso, centrado em $g=2,07$ (3272 G na Figura 2), pode estar associado à presença de íons prata. Os íons $\mathrm{Ag}^{2+} \mathrm{e}$ os átomos $\mathrm{Ag}^{0}$, de configurações eletrônicas $4 \mathrm{~d}^{9}$ e $4 \mathrm{~d}^{10} 5 \mathrm{~s}^{1}$, respectivamente, são paramagnéticos, mas o íon $\mathrm{Ag}^{+}$, de configuração eletrônica $4 \mathrm{~d}^{10}$, não é paramagnético e, portanto, não gera espectro de RPE. Garnica-Romo

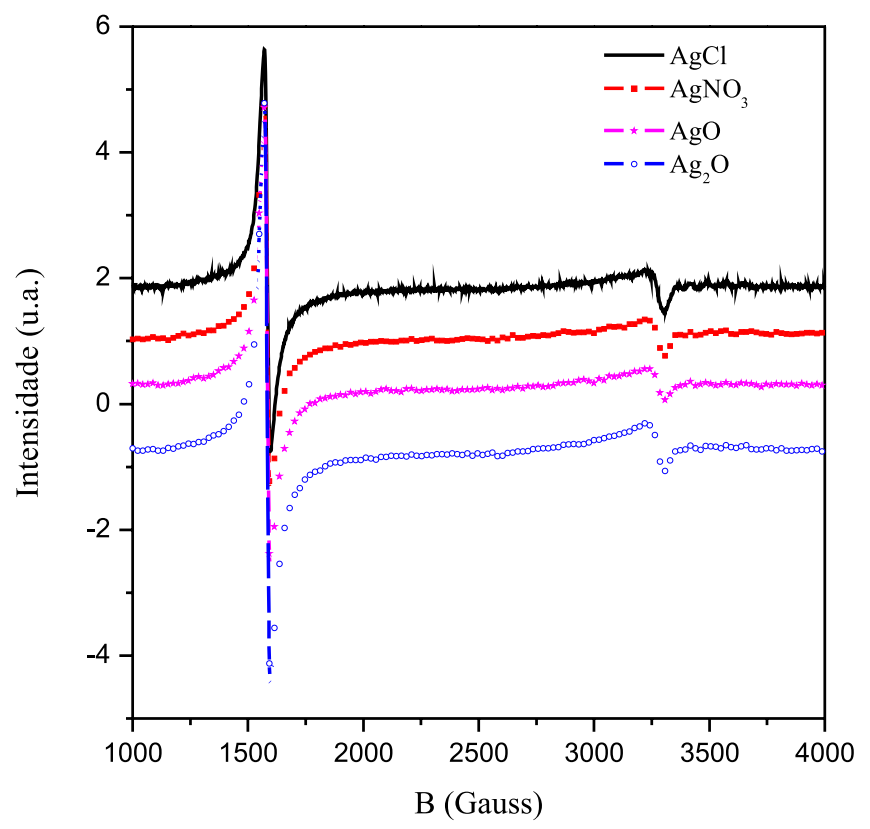

Figura 2. Curvas de RPE dos vidros sem tratamento térmico contendo diferentes precursores de prata e obtidos a $-258^{\circ} \mathrm{C}$ e col. identificaram sinais espectrais em $g$ » 1,95, em vidros de $\mathrm{SiO}_{2}$ dopados com $\mathrm{AgNO}_{3}$ tratados termicamente, como resultantes de elétrons desemparelhados em nanopartículas de prata. ${ }^{18}$ Mitrikas e col. também associaram o sinal de RPE observado em $\mathrm{g}=2,0$, assim como as curvas de recuperação do eco de spin eletrônico, em matrizes de $\mathrm{SiO}_{2}$ dopadas com $\mathrm{AgNO}_{3}$ com a presença de nanopartículas de prata. ${ }^{19}$ As pequenas diferenças observadas nos valores de $g$ com relação ao valor da prata metálica $(g=1,9827)$ têm sido atribuídas a efeitos quânticos nos agregados de prata. $^{20}$

Com o intuito de avaliar a influência dos precursores de prata nas propriedades estruturais das amostras vítreas, estas foram estudadas usando espectroscopia Raman. Nenhuma variação significativa foi observada nos espectros em função da natureza do composto de prata adicionado (Figura 1Sa). Devido à semelhança dos espectros, estes são mostrados somente no material suplementar, disponível on-line.

Da mesma maneira, as propriedades ópticas analisadas por UVVis não sofrem nenhuma variação nesta região. Nota-se também que a adição dos diferentes precursores de prata não causa mudanças significativas no gap óptico dos vidros, como pode ser visualizado na Figura 2S. Os valores de índices de refração dos vidros foram medidos para $\lambda=632 \mathrm{~nm}$ e apresentaram valores iguais $(2,072)$ para a amostra contendo $\mathrm{AgCl}, \mathrm{AgNO}_{3}$ e $\mathrm{Ag}_{2} \mathrm{O}$, enquanto o valor obtido para a amostra contendo AgO foi 2,070. Este conjunto de resultados mostra que, independentemente da natureza química do composto de prata utilizado, não há variação significativa nos valores de índice de refração dos vidros.

\section{Caracterização dos vidros após o tratamento térmico}

Todas as amostras foram tratadas termicamente em atmosfera ambiente por 5 e $10 \mathrm{~h}$ a $480{ }^{\circ} \mathrm{C}$, portanto, acima da temperatura da transição vítrea. A influência do tratamento térmico também foi estudada por análise térmica. Na Figura 1b podemos observar que, para os vidros tratados durante $5 \mathrm{~h}$, os valores de $\mathrm{Tg}$ permanecem constantes para as amostras contendo $\mathrm{AgCl}$ e $\mathrm{AgNO}_{3}$, enquanto que uma ligeira diminuição foi observada para as amostras contendo os óxidos. Por outro lado, os valores de Tx e do parâmetro Tx-Tg, diminuem para todas as amostras. Na Figura 1c verificamos que o aumento do tratamento térmico $(10 \mathrm{~h})$ não altera significativamente os valores das temperaturas características dos vidros em relação ao tratamento realizado durante $5 \mathrm{~h}$. Uma característica que se torna mais evidente após o tratamento térmico é a separação dos picos de cristalização presentes nas amostras contendo $\mathrm{AgCl}$ e $\mathrm{AgNO}_{3}$. Notou-se uma grande semelhança entre as curvas, sugerindo que tanto o processo de cristalização como as fases cristalinas formadas foram semelhantes. Seguindo o mesmo raciocínio, as curvas dos vidros contendo os óxidos também se comportam de mesma maneira.

As amostras tratadas por 5 e $10 \mathrm{~h}$ também foram estudadas utilizando espectroscopia Raman e observou-se que, mesmo após o tratamento térmico, os espectros não apresentam variações significativas nas energias das bandas existentes em relação as amostras não 
tratadas. Por outro lado, as curvas de absorção na região do UV-Vis para as amostras contendo $\mathrm{AgCl}$ e $\mathrm{AgNO}_{3}$ apresentam diferenças significativas quando comparadas com as amostras sem tratamento térmico. Como podemos observar na Figura 3, após o tratamento térmico de $5 \mathrm{~h}$, as amostras contendo cloreto e nitrato de prata apresentam bandas de absorção centradas em 552 e $545 \mathrm{~nm}$, respectivamente, enquanto nenhuma mudança significativa na absorção foi observada para as amostras contendo os óxidos. O tratamento térmico durante $10 \mathrm{~h}$ aumenta ligeiramente a intensidade das bandas da amostra contendo cloreto de prata, como mostrado no inserto da Figura 3, porém, novamente, não provoca nenhuma mudança nos espectros das amostras contendo óxidos.

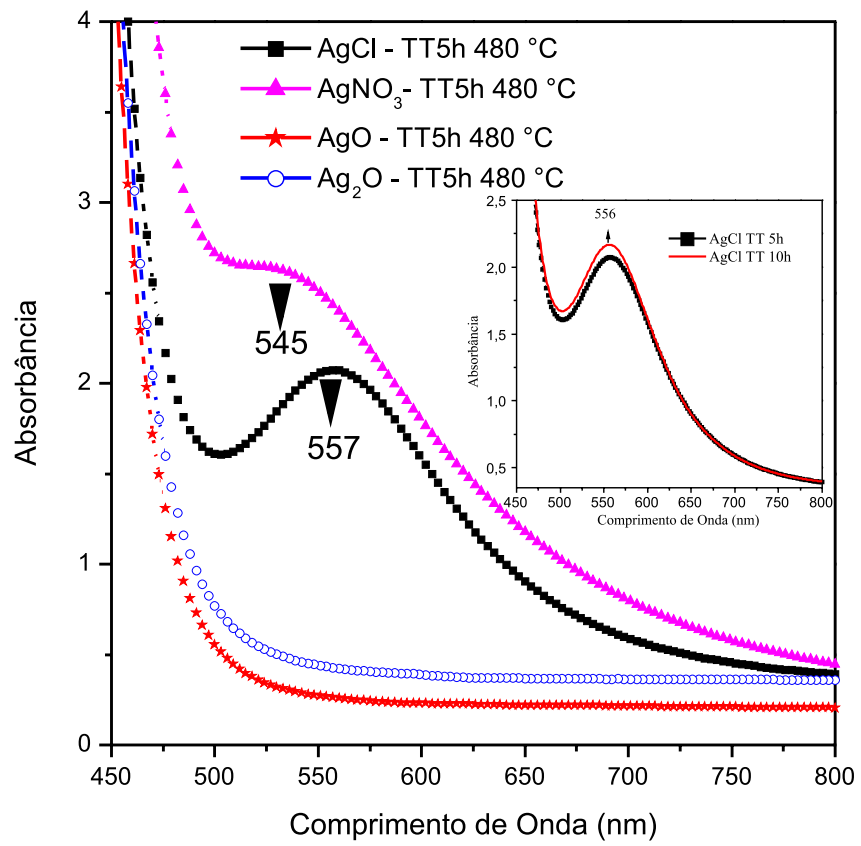

Figura 3. Espectros de absorção na região do UV-Vis das amostras tratadas termicamente a $480^{\circ} \mathrm{C}$ durante 5 e $10 \mathrm{~h}$. Inserto: Diferença na intensidade da banda SPR para as amostras contendo $\mathrm{AgCl}$, tratadas por diferentes períodos $a 480^{\circ} \mathrm{C}$

As bandas de absorção podem ser atribuídas à ressonância de plasmons superficiais (SPR) decorrentes do crescimento dos clusters de prata e culminando na formação das nanopartículas metálicas no interior dos vidros. Este fenômeno é bem conhecido e vem sendo bastante explorado nos últimos anos devido à possibilidade de sua utilização em vidros, para aumentar a emissão de terras raras na região do infravermelho, visando a aplicações em amplificadores para telecomunicações. ${ }^{9}$ Resultados recentes de obtenção de nanopartículas em vidros óxidos de metais pesados ${ }^{21}$ mostram que a energia da banda SPR depende de vários fatores tais como o tamanho da nanopartícula, sua geometria e o valor do índice de refração do dielétrico (neste caso, da matriz vítrea). O aumento do tamanho da partícula e/ou do índice de refração do vidro pode ser responsável pela diminuição da energia da SPR. A intensidade da banda pode ser relacionada, principalmente, ao número de partículas. Assim, atribuímos o ligeiro aumento na intensidade da banda SPR, observado para amostra tratada $10 \mathrm{~h}$, ao aumento do número de partículas. A confirmação da presença das nanopartículas nos vidros contendo $\mathrm{AgCl}$ e $\mathrm{AgNO}_{3}$ foi obtida por MET a partir das amostras tratadas durante $10 \mathrm{~h}$ e pode ser observada nas Figuras 4a e 4b, respectivamente. Como insertos nas Figuras 4a e 4b são mostradas as curvas de distribuição média do tamanho das nanopartículas que, podemos notar, são bastante semelhantes, apresentando os valores de 3,2 $\mathrm{nm}$ para a amostra contendo $\mathrm{AgCl}$ e 3,1 nm para amostra contendo $\mathrm{AgNO}_{3}$. Os valores dos índices de refração foram novamente medidos para estas duas amostras após o tratamento térmico de $10 \mathrm{~h}$. Os resultados obtidos para as amostras contendo $\mathrm{AgCl}$ e $\mathrm{AgNO}_{3}$ apontam um aumento de $\mathrm{n}$ (em $\lambda=632$ $\mathrm{nm}$ ) passando de 2,072 (para as amostra sem tratamento) para 2,080. $\mathrm{O}$ aumento de $\mathrm{n}$ pode ser atribuído ao aumento da polarizabilidade advindo da presença das nanopartículas metálicas. Entretanto, como $\mathrm{o}$ aumento de $\mathrm{n}$ foi igual para as duas amostras, a diferença de energia entre as bandas SPR deve ser atribuída à diferença do tamanho médio das partículas como foi observado por microscopia.

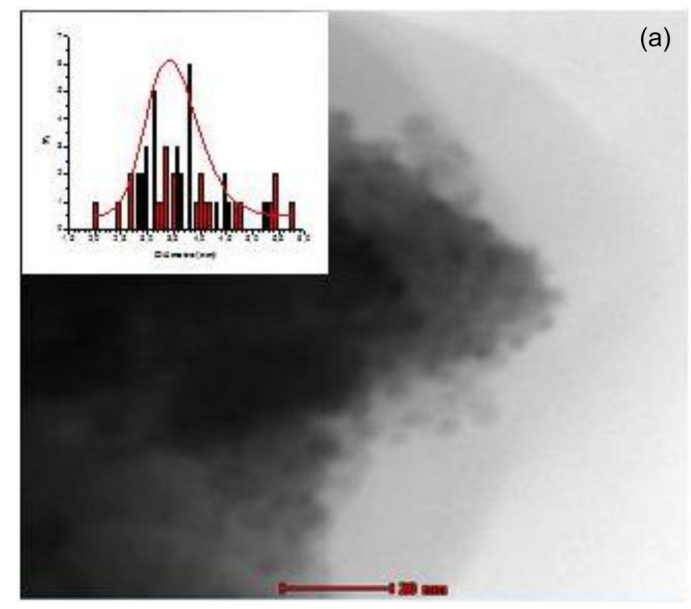

(b)

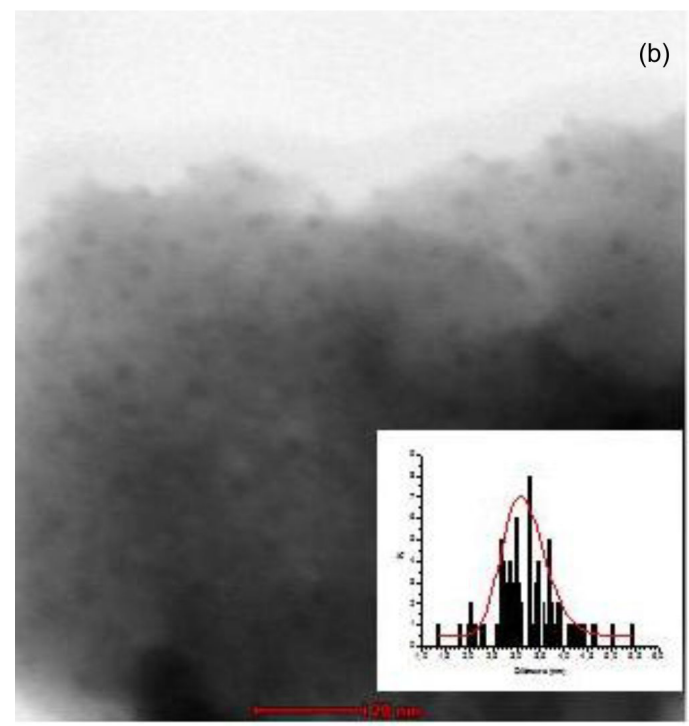

Figura 4. Microscopias eletrônica de transmissão das amostras contendo $\mathrm{AgCl}($ a $)$ e $\mathrm{AgNO}_{3}$ (b) tratadas durante 10 h. Insertos: Curvas de distribuição do tamanho médio de partículas obtidos para cada microscopia

Na sessão anterior, discutimos a respeito da existência de prata metálica dispersa no vidro, assim, podemos afirmar agora que o tratamento térmico acima de $\mathrm{Tg}$ para as amostras contendo $\mathrm{AgCl}$ e $\mathrm{AgNO}_{3}$ favorece a migração dos átomos de prata levando à formação das nanopartículas. Dessa maneira, observamos que as propriedades (térmicas e ópticas) das duas amostras são muito parecidas, sugerindo que, após o processo de fusão, as composições dos vidros sejam semelhantes.

As amostras contendo $\mathrm{Ag}_{2} \mathrm{O}$ e $\mathrm{AgO}$ apresentaram propriedades distintas dos vidros discutidos acima. Além de não mudarem de coloração após o tratamento térmico de 10 h, também apresentaram 
comportamento diferente na análise térmica e, finalmente, não possuem nanopartículas, como foi verificado por MET. Uma possível explicação para esse fato é que $\mathrm{o} \mathrm{Ag}_{2} \mathrm{O}$ e o $\mathrm{AgO}$ podem não ter sido totalmente decompostos durante o processo de fusão dos vidros, permanecendo, em grande parte como $\mathrm{Ag}_{2} \mathrm{O}$ e, possivelmente, fazendo parte das estruturas dos vidros. A presença dos óxidos aumentou a estabilidade vítrea da composição $\mathrm{SbPO}_{4}-\mathrm{WO}_{3}-\mathrm{PbO}$ sugerindo que unidades do tipo -Ag-O-Ag-O- podem estar ligadas à estrutura que compõe o esqueleto da matriz vítrea e que, mesmo após o tratamento térmico acima de $\mathrm{Tg}$, não ocorre a difusão dos átomos de prata e a consequente formação de uma estrutura organizada, a qual poderia ser metálica ou mesmo do óxido. Estas conclusões foram baseadas nos resultados discutidos previamente nas referências,,$^{22,23}$ as quais discutem dados obtidos para sistemas vítreos à base de sílica ${ }^{22} \mathrm{e}$ outros oxidos ${ }^{23}$ e onde foi identificada a presença de prata na forma de $\mathrm{Ag}_{2} \mathrm{O}$, mesmo com a síntese dos vidros sendo realizada acima de $950{ }^{\circ} \mathrm{C}$. Neste contexto, e considerando os resultados obtidos em nosso trabalho, acreditamos que nossas conclusões estão de acordo com os dados relatados por estes autores.

Por outro lado, a hipótese mais provável para a ausência das nanopartículas metálicas de prata deve estar relacionada à temperatura usada para o tratamento térmico. Devido ao maior valor de estabilidade térmica determinado para as amostras contendo os óxidos, a temperatura escolhida para o tratamento térmico (480 $\left.{ }^{\circ} \mathrm{C}\right)$ pode não ser suficientemente alta para induzir a difusão da prata pela estrutura do vidro. Entretanto, salientamos que o valor da temperatura de tratamento térmico foi escolhida com base nos valores de $\mathrm{Tg}$ dos vidros.

A presença das nanopartículas metálicas nos vidros pode ser explorada de diversas maneiras, sendo que duas aplicações se destacam. A primeira está relacionada às propriedades não lineares dos vidros óxidos de metais pesados, dos quais a formação das nano-vitrocerâmicas pode melhorar ainda mais as propriedades e resultar na fabricação de dispositivos totalmente ópticos, como vem sendo proposto em vários trabalhos. ${ }^{21,24}$ Outra aplicação é a preparação de amplificadores ópticos contendo, simultaneamente, nanopartículas metálicas e íons terras raras, tais como $\mathrm{Er}^{3+} \mathrm{e} \mathrm{Tm}^{3+}$. Em princípio, dependendo da matriz vítrea e da natureza das nanopartículas metálicas, pode-se excitar o vidro na banda SPR e, posteriormente, os sólitons gerados transferem a energia para as terras raras de uma maneira mais efetiva que a excitação direta, fazendo com que a eficiência de emissão no infravermelho aumente. Isto permitiria a preparação de dispositivos amplificadores mais eficazes que os disponíveis. Atualmente, estamos explorando ambos os efeitos em nosso laboratório e os resultados preliminares são bem animadores, tanto para o aumento da não linearidade como para o estudo da transferência de energia entre a nanopartícula e as terras raras em matrizes de óxidos de metais pesados.

\section{CONCLUSÕES}

Observamos que a escolha dos compostos de prata utilizados para a preparação de vitrocerâmicas contendo nanopartículas metálicas é extremamente importante e que, dependendo da natureza do composto e das condições de tratamento térmico, pode não ocorrer a formação das nano-vitrocerâmicas.

Nano-vitrocerâmicas foram preparadas usando como reagentes de partida $\mathrm{AgCl}$ e $\mathrm{AgNO}_{3}$, os quais são decompostos em átomos de prata metálica durante o processo de fusão, que se aglomeram sob tratamento térmico acima de $\mathrm{Tg}$.
Por outro lado, a adição de $\mathrm{Ag}_{2} \mathrm{O}$ e $\mathrm{AgO}$ não levou à formação de vitrocerâmicas, sugerindo que a temperatura e/ou tempo de tratamento térmico do vidro não foram suficientes para induzir a difusão dos átomos de Ag e, como consequência, não ocorreu formação de nanopartículas metálicas.

\section{MATERIAL SUPLEMENTAR}

Está disponível em http://quimicanova.sbq.org.br, na forma de arquivo PDF, com acesso livre e é constituído pelas Figuras $1 \mathrm{~S}$ e 2S.

\section{AGRADECIMENTOS}

Os autores agradecem ao CNPq, CAPES e FAPESP (CEPID 2011/51368-2) pelo auxílio financeiro e ao Prof. Dr. S. J. L. Ribeiro do LAMF, IQ - UNESP de Araraquara pelas medidas de análise térmica, espectroscopias Raman e M-Lines.

\section{REFERÊNCIAS}

1. Varma, K. B. R.; Paramesh, G.; Int. J. Appl. Glass Sci. 2011, 2, 235.

2. Nikonorov, N. V.; Sidorov, A. I.; Tsekhomskii, V. A.; Nashchekin, A. V.; Usov, O. A.; Podsvirov, O. A.; Poplevkin, S. V.; Pis'ma v Zh. Tekh. Fiz. 2009, 35, 35.

3. Vostokov, A. V.; Verzin, I. A.; Ignat'ev, A. I.; Podsvirov, O. A.; Sidorov, A. I.; Opt. Spektrosk. 2010, 109, 407.

4. Lin, C. H.; Jiang, L.; Chai, Y. H.; Xiao, H.; Chen, S. J.; Tsai, H. L.; Opt. Express. 2009, 17, 21582.

5. Varshneya, A. K.; Fundamentals of Inorganic Glasses, $2^{\text {nd }}$ ed., Society of Glass Technology: Sheffield, 2006.

6. Lee, P. C.; Meise, D.; J. Phys. Chem. 1982, 86, 3391.

7. Amekura, H.; Takeda, Y.; Kishimoto, N.; Thin Sol. Films. 2004, 464, 268.

8. Speranza, G.; Minati, L.; Chiasera, A.; Ferrari, M.; Righini, G. C.; Ischia, G.; J. Phys. Chem. 2009, 113, 4445.

9. Eichelbaum, B. M.; Rademann, K.; Adv. Funct. Mater. 2009, 19, 2045.

10. Homola, J.; Yee, S. R.; Gauglitz, G.; Sens. Actuators, B 1999, 54, 3.

11. Nalin, M.; Messaddeq, Y.; Ribeiro, S. J. L.; Poulain, P.; Briois, V.; Brunklaus, G.; Rosenhahn, C.; Mosel, B. D.; Eckert, H.; J. Mater. Chem. 2004, 14, 3398.

12. Bregadiolli, B. A.; Souza, E. R.; Sigoli, F. A.; Caiut, J. M. A.; Alencar, M. A. S.; Benedetti, A. V.; Nalin, M.; Quim. Nova 2012, 35, 755.

13. Navarro, J. M. F.; El Vidrio, $3^{\text {a }}$ ed., CSIC: Madri, 2003, p. 471.

14. Shannon, R. S.; Trans. Faraday Soc. 1964, 60, 1902.

15. Griscom, D. L.; J. Non-Cryst. Solids 1980, 40, 211.

16. Gan, F.; Liu, H.; J. Non-Cryst. Solids 1987, 95, 61.

17. Ardelean, I.; Toderas, M.; Horea, C.; Filip, S.; J. Optoelectron. Adv. Mater. 2008, 10, 243.

18. Garnica-Romo, M. G.; Limón, J. M. Y.; Gonzales-Hernández, J.; Ramírez-Bon, R.; Ramírez-Rosales, D.; Zamorano-Ulloa, R.; Tirado-Guerra, S.; J. Sol-Gel Sci. Technol. 2002, 24, 105.

19. Mitrikas, G.; Trapalis, C. C.; Kordas, G.; J. Chem. Phys. 1999, 111, 8098.

20. Ma, S. K.; Lue, J. T.; Solid State Commun. 1996, 97, 979.

21. Svedberg, F.; Li, Z.; Xu, H.; Kall, M.; Nano Lett. 2006, 6, 2639.

22. Nikonorov, N. V.; Sidorov, A. I.; Tsekhomskii, V. A. Em Silver Nanoparticles; Perez, D. P., eds; InTech, 2010, cap. 10.

23. Chvatal T. L.; US Patent, $n^{\circ}$ 3.853568, 1974.

24. Kassab, L. R. P.; Silva, D. S.; Almeida, R.; Araujo, C. B.; Appl. Phys. Lett. 2009, 94, 101912. 


\section{INFLUÊNCIA DOS PRECURSORES DE PRATA NO CRESCIMENTO DE NANOPARTÍCULAS METÁLICAS EM VIDROS ÓXIDOS DE METAIS PESADOS}

Paula S. Valle, Murilo Montesso e Marcelo Nalin*

Departamento de Química - Universidade Federal de São Carlos, CP 676, 13565-905 São Carlos - SP, Brasil

José Pedro Donoso, Igor D.A. Silva e Claudio J. Magon

Instituto de Física de São Carlos - Universidade de São Paulo, CP 369, 13560-970 São Carlos - SP, Brasil

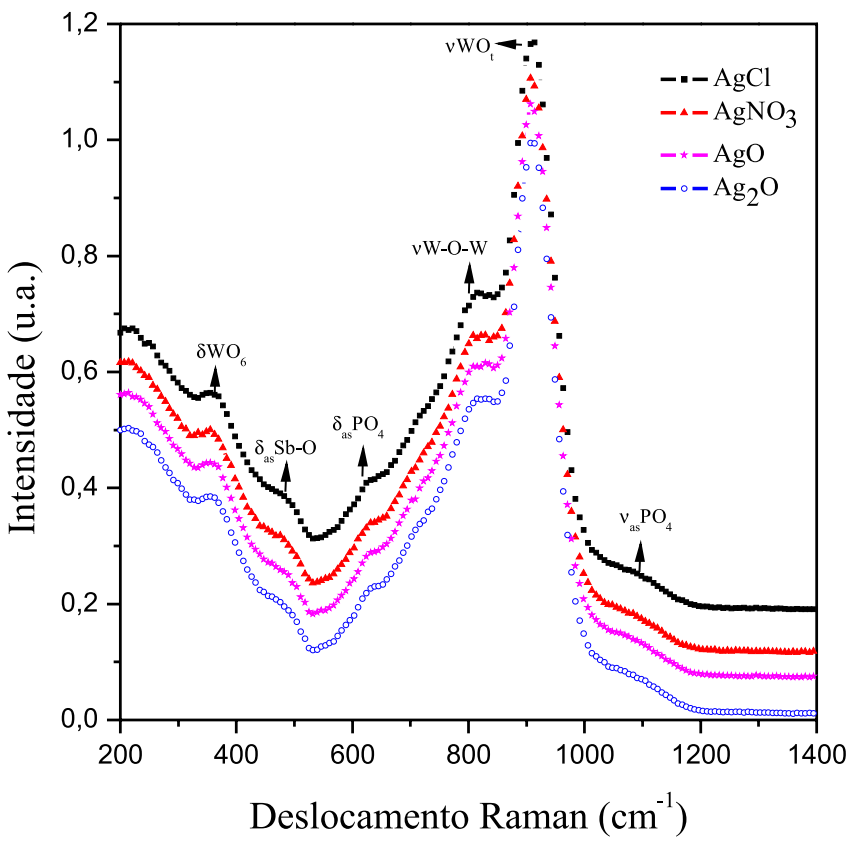

Figura 1S. Espectros Raman obtidos das amostras vitreas, sem tratamento térmico, contendo diferentes precursores de prata. Os dados obtidos para as amostras tratadas durante 5 e $10 \mathrm{~h}$ não são mostrados pois são sobreponíveis àqueles das amostras sem tratamento. As atribuições das bandas estão indicadas no gráfico

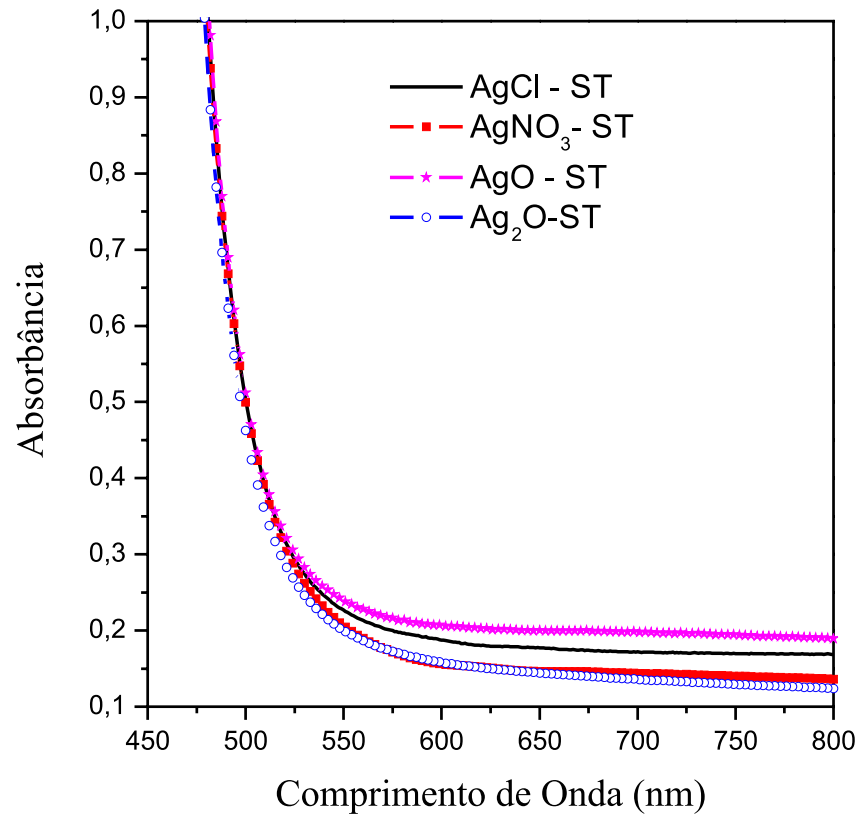

Figura 2S. Curvas de absorção na região do visível das diferentes composições de vidro para as amostras sem tratamento térmico 